\title{
Rayleigh-Taylor instabilities with sheared magnetic fields in partially ionised plasmas
}

\author{
M. S. Ruderman ${ }^{1,2}$, I. Ballai ${ }^{1}$, E. Khomenko ${ }^{3,4}$, and M. Collados ${ }^{3,4}$ \\ ${ }^{1}$ Solar Physics and Space Plasma Research Centre (SP $\left.{ }^{2} \mathrm{RC}\right)$, University of Sheffield, Hicks Building, Hounsfield Road, \\ Sheffield S3 7RH, UK \\ e-mail: m.s.ruderman@sheffield.ac.uk \\ 2 Space Research Institute (IKI) Russian Academy of Sciences, Moscow 117810, Russia \\ 3 Instituto de Astrofísica de Canarias, 38205, C/vía Láctea, s/n, 38205 La Laguna, Tenerife, Spain \\ ${ }^{4}$ Departamento de Astrofísica, Universidad de La Laguna, 38205 La Laguna, Tenerife, Spain
}

Received 8 July 2017 / Accepted 24 September 2017

\begin{abstract}
Aims. In the present study we investigate the nature of the magnetic Rayleigh-Taylor instability appearing at a tangential discontinuity in a partially ionised plasma when the effect of magnetic shear is taken into account.

Methods. The partially ionised character of the plasma is described by the ambipolar diffusion in the induction equation. The dynamics of the plasma is investigated in a single-fluid approximation. After matching the solutions on both sides of the interface we derive a dispersion equation and calculate the instability increment using analytical methods for particular cases of parameters, and numerical investigation for a wide range of parameters.

Results. We calculated the dependence of the instability increment on the perturbation wavenumber. We also calculated the dependence of the maximum instability increment on the shear angle of the magnetic field for various values of the ionisation degree.

Conclusions. Our results show that the Rayleigh-Taylor instability becomes sensitive to the degree of plasma ionisation only for plasmas with small values of plasma beta and in a very weakly ionised state. Perturbations are unstable only for those wavenumbers that are below a cut-off value.
\end{abstract}

Key words. magnetohydrodynamics (MHD) - instabilities - magnetic fields - Sun: corona

\section{Introduction}

One of the key questions related to magnetic structures in space plasmas is their stability, that is the analysis of the evolution of perturbations over time. Instabilities are ubiquitous in space plasmas as they can provide the dissipation and transport of momentum over large scales.

The magnetic Rayleigh-Taylor (MRT) instability operates in a variety of astrophysical systems. For example, it manifests itself in buoyant magnetised bubbles identified in clusters of galaxies (Robinson et al. 2004; Jones \& De Young 2005; O'Neil et al. 2009), in shells of young supernova remnants (Jun et al. 1995; Jun \& Norman 1996), and at the interface between an expanding pulsar wind nebula and its surrounding supernova remnant (Bucciantini et al. 2004)

The MRT instability is also very important in applications to solar physics. Isobe et al. $(2005,2006)$ proposed that the MRT instability is a possible cause of the filamentary structure in mass and current density in the emerging flux regions. Ryutova et al (2010) suggested that several dynamic processes taking place in prominences are most probably related to the MRT instabilities. Hilier et al. (2011, 2012a,b) have performed three-dimensional magnetohydrodynamic simulations to investigate the non-linear evolution of the Kippenhahn-Shlüter prominence model due to the MRT instability.

The MRT instability may also affect magnetic threads in solar prominences. The threads are parts of magnetic tubes filled with the colder plasma and with a high density contrast with respect to the coronal plasma. They are quite thin, of the order of $100 \mathrm{~km}$, aligned with the magnetic field and, in many cases, they seem to lie horizontally with respect to the photosphere. Because of their low temperature the thread plasma is only partially ionised. The MRT instability in partially ionised plasmas has been studied both analytically (Díaz et al. 2014) and numerically (Khomenko et al. 2014) under an assumption that the magnetic field both in the dense prominence thread and in the surrounding hot plasma has the same direction. However, observations show (Leroy et al. 1984; Bommier et al. 1994) that the angle between the magnetic field vector and the prominence long axis can be as large as $53^{\circ} \pm 15^{\circ}$. In addition, the magnetic field in the corona can also be tilted with respect to the longitudinal axis of the prominence. This is why in a realistic model the magnetic field at the interface between the prominence and corona has a shear, perfectly justifying our present analysis.

Observations show that the threads have short lifetimes, typically of the order of only $10 \mathrm{~min}$. These observations inspired Terradas et al. (2012) to propose that the magnetic RayleighTaylor instability could be responsible for this phenomenon. They considered a very simple model. The thread was assumed to be a Cartesian slab permeated by a horizontal magnetic field that has the same direction inside the thread and in the surrounding hot plasma. A drawback of this model is that the instability growth rate is unbounded. Perturbations with an arbitrary wavelength propagating perpendicular to the magnetic field are unstable, and the perturbation increment tends to infinity when the wavelength tends to zero. 
Ruderman et al. (2014) improved the model suggested by Terradas et al. (2012) including the magnetic shear. They studied the MRT instability of both a single magnetic interface and a slab. In particular, they found that the maximum increment is inversely proportional to the angle between the magnetic field direction inside the thread and in the external plasma. Assuming that the thread lifetime is equal to the inverse of the maximum increment they managed to estimate the shear angle. Ruderman (2015) studied the effect of flow in a thread on the estimation of the shear angle and found that this effect is minor. Ruderman (2017) investigated the MRT instability in a compressible plasma and found that the effect of compressibility on the estimation of the shear angle is also weak.

Terradas et al. (2012), Ruderman et al. (2014), and Ruderman $(2015,2017)$ used the approximation of ideal plasmas to study the MRT instability. However, as we have already mentioned, due to its low temperature the prominence plasma is only partially ionised. Díaz et al. (2014) showed that in such a plasma ambipolar diffusion has to be taken into account. In this article we investigate the effect of ambipolar diffusion on the MRT instability with sheared magnetic field. Even in the approximation of ideal plasmas the dispersion equation describing the MRT instability in a compressible plasma is very complex and can only be solved numerically. The account of ambipolar diffusion makes the dispersion equation even more complex. Using the incompressible plasma approximation enormously simplifies the analysis and enables to study the problem analytically. Unfortunately, in this approximation the effect of ambipolar diffusion disappears, hence we need to study the effect of ambipolar diffusion in a compressible plasma.

The paper is organised as follows: in Sect. 2, we formulate the problem and introduce the main equations and boundary conditions. Section 3 is devoted to the derivation of the dispersion equation for perturbations of the interface. Analytical and numerical solutions are found in Sect. 4. Finally, our results are summarised and discussed in Sect. 5.

\section{Problem formulation}

Our study focuses on the stability of a tangential discontinuity also called a magnetic interface in an inviscid infinitely conducting plasma, where the only non-ideal process that we take into account is ambipolar diffusion. The linearised equations describing the plasma motion in the presence of ambipolar diffusion in the single-fluid approximation have been derived by Díaz et al. (2014) and they can be given as

$$
\begin{aligned}
& \frac{\partial \rho}{\partial t}+\rho_{0} \nabla \cdot \boldsymbol{u}=0, \\
& \rho_{0} \frac{\partial \boldsymbol{u}}{\partial t}=-\nabla p+\frac{1}{\mu_{0}}(\nabla \times \boldsymbol{b}) \times \boldsymbol{B}+\rho \boldsymbol{g}, \\
& \frac{\partial \boldsymbol{b}}{\partial t}=\nabla \times\left(\boldsymbol{u} \times \boldsymbol{B}+\frac{\eta_{\mathrm{A}}}{B^{2}}[(\nabla \times \boldsymbol{b}) \times \boldsymbol{B}] \times \boldsymbol{B}\right), \\
& \frac{\partial p}{\partial t}=-\gamma p_{0} \nabla \cdot \boldsymbol{u} .
\end{aligned}
$$

In the above equations $\boldsymbol{u}$ is the plasma velocity, $\boldsymbol{b}$ the magnetic field perturbation, $\boldsymbol{g}$ the gravity acceleration, $\boldsymbol{B}$ the equilibrium magnetic field, $\rho_{0}$ and $p_{0}$ the equilibrium density and pressure, $\rho$ and $p$ the density and pressure perturbation, $\mu_{0}$ magnetic permeability of free space, $\eta_{\mathrm{A}}$ the coefficient of ambipolar diffusion, and $\gamma$ the ratio of specific heats. The coefficient of ambipolar diffusion is given by

$\eta_{\mathrm{A}}=\frac{m_{\mathrm{p}} \xi_{\mathrm{n}} c_{\mathrm{A}}^{2}}{4 \sigma_{\mathrm{in}}\left(1-\xi_{\mathrm{n}}\right)} \sqrt{\frac{\pi\left(2-\xi_{\mathrm{n}}\right)}{\rho_{0} p_{0}}}$,

where $m_{\mathrm{p}}$ is the proton mass, $\xi_{\mathrm{n}}$ the ionisation degree defined as the ratio of the number density of neutrals and the total number density (neutrals plus ions), $\sigma_{\text {in }} \approx 5 \times 10^{-19} \mathrm{~m}^{2}$ the collisional cross-section for proton-neutral collisions assuming that the neutrals are the hydrogen atoms, and $c_{\mathrm{A}}$ is the Alfvén speed defined as

$c_{\mathrm{A}}^{2}=\frac{B^{2}}{\mu_{0} \rho_{0}}$.

It should be noted that when Díaz et al. (2014) derived the system of Eqs. (1)-(4), they assumed that all the equilibrium quantities are constant. The validity of this assumption is discussed in detail by, e.g. Ruderman (2017). In what follows we also need the expression for the generalised Ohm's law, where ambipolar diffusion is taken into account. In the linearised form the Ohm's law reads

$\boldsymbol{E}^{\prime} \equiv \boldsymbol{E}+\boldsymbol{u} \times \boldsymbol{B}=\frac{\eta_{\mathrm{A}}}{B^{2}} \boldsymbol{B} \times[(\nabla \times \boldsymbol{b}) \times \boldsymbol{B}]$,

where $\boldsymbol{E}$ is the electric field. This equation immediately follows from Eq. (3) if we recall the relation between the time derivative of the magnetic field and the curl of the electrical field.

In our analysis we consider the same equilibrium configuration as in Ruderman et al. (2014) and Ruderman (2017). Our working model consists of two semi-infinite regions separated by the $x y$-plane in Cartesian coordinates $x, y, z$ with the $z$-axis in the vertical direction (see Fig. 1). The plasma density and background magnetic fields are constant in the two regions, and are given by

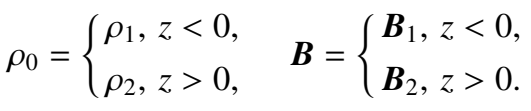

The background magnetic field in both regions is assumed to be parallel to the $x y$-plane. The major difference between the equilibrium configuration used here and that used by Díaz et al. (2014) is that here we consider a magnetic shear, while Díaz et al. (2014) assumed that the equilibrium magnetic field is the same at the two sides of the interface. In Fig. 1 the angle $\alpha$ denotes the angle between the direction of the two magnetic fields (i.e. a measure of the magnetic shear), while the angle $\phi$ determines the direction of the perturbation wave vector with respect to the direction of the magnetic field in the prominence.

Since we are dealing with a tangential discontinuity, the equilibrium total plasma pressure must be continuous at the interface, so

$p_{1}+\frac{B_{1}^{2}}{\mu_{0} \rho_{1}}=p_{2}+\frac{B_{2}^{2}}{\mu_{0} \rho_{2}}$.

The equilibrium magnetic field is discontinuous at $z=0$. In general, the magnetic field discontinuity cannot exist in a plasma with ambipolar diffusion. However, the only exception is when ambipolar diffusion operates only on one side of the discontinuity. Therefore, in what follows we only consider ambipolar diffusion in the plasma region above the discontinuity, while we assume that the plasma dynamics is described by the ideal MHD equations in the region below the discontinuity. This set-up is a 


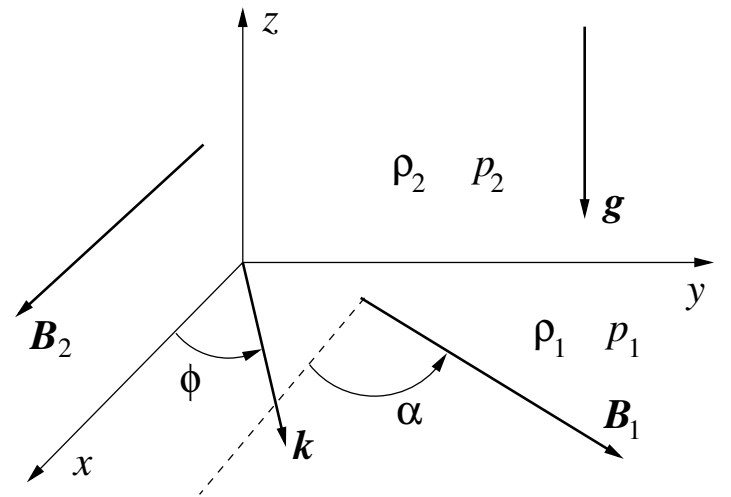

Fig. 1. Sketch of the equilibrium. The interface at $z=0$ separates the fully ionised solar corona (region 1) and the partially ionised prominence (region 2).

very viable assumption because the plasma below the discontinuity is almost fully ionised, meaning that $\xi_{\mathrm{n}} \approx 0$. Then it follows from Eq. (5) that $\eta_{\mathrm{A}}$ is very small and ambipolar diffusion can be neglected.

Equations (1)-(4) must be supplemented with the boundary conditions at $z=0$. Let us define the jump of function $f(z)$ across the interface as

$\llbracket f \rrbracket=\lim _{z \rightarrow+0}[f(z)-f(-z)]$.

The first boundary condition is the kinematic boundary condition that expresses the continuity of the normal component of plasma displacement at the interface. In the absence of equilibrium flow this condition reduces in the linear approximation to a very simple form:

$\llbracket u_{z} \rrbracket=0$.

The second boundary condition is the dynamic boundary condition expressing the continuity of the total pressure. It is written as

$\llbracket p_{0}+p+\frac{(\boldsymbol{B}+\boldsymbol{b})^{2}}{2 \mu_{0}} \rrbracket=0 \quad$ at $\quad z=h(t, x, y)$

where $z=h(t, x, y)$ is the equation of the perturbed surface of the discontinuity. We can linearise this equation, and using the Taylor expansion of $p_{0}$ near $z=0$ and the equation defining the equilibrium pressure $\left(\mathrm{d} p_{0} / \mathrm{d} z=-g \rho_{0}\right)$, we eventually obtain

$\llbracket P-g \rho_{0} h \rrbracket=0$ at $z=0$,

where

$P=p+\frac{\boldsymbol{b} \cdot \boldsymbol{B}}{\mu_{0}}$

is the perturbation of the total pressure (kinetic plus magnetic). Differentiating this equation with respect to time and using the equation $u_{z}=\partial h / \partial t$ valid in the linear approximation at $z=0$ to eliminate $h$, we finally arrive at

$\llbracket g \rho_{0} u_{z}-\frac{\partial P}{\partial t} \rrbracket=0$

The continuity of the normal component of the plasma velocity and the total pressure perturbation are the only two boundary conditions that must be imposed at a tangential discontinuity in ideal MHD. However, since we consider ambipolar diffusion, we need one more boundary condition that can be obtained using the continuity of tangential component of the electric field calculated in the instantaneous reference frame, where a particular infinitesimal volume of plasma is at rest, i.e. $\boldsymbol{E}_{\tau}^{\prime}=\boldsymbol{E}^{\prime}-\boldsymbol{e}_{z}\left(\boldsymbol{e}_{z} \cdot \boldsymbol{E}^{\prime}\right)$ with $\boldsymbol{e}_{z}$ being the unit vector in the $z$-direction. Then, using Eq. (7) and taking into account that $\eta_{\mathrm{A}}=0$ for $z<0$ we obtain the additional boundary condition that has to be satisfied at $z=0$ in the form

$\left(B_{2}^{2} \mathbf{I}-\boldsymbol{B}_{2} \boldsymbol{B}_{2}-B_{2}^{2} \boldsymbol{e}_{z} \boldsymbol{e}_{z}\right) \cdot \nabla \times \boldsymbol{b}_{2}=0$,

where $\mathbf{I}$ is the unit tensor, $\boldsymbol{e}_{z} \boldsymbol{e}_{z}$ denotes the dyadic product of two vectors $\boldsymbol{e}_{z}$, and the subscript " 2 " indicates a quantity defined for $z>0$.

\section{Derivation of the dispersion equation}

In order to derive the dispersion equation for waves propagating along the magnetic interface, we performed a Fourier analysis of the perturbations of all quantities and take them proportional to $\exp [\mathrm{i}(\boldsymbol{k} \cdot \boldsymbol{r}-\omega t)]$, where $\boldsymbol{k}=\left(k_{x}, k_{y}, 0\right)$ and $\boldsymbol{r}=(x, y, z)$. Then, writing $\boldsymbol{u}$ and $\boldsymbol{b}$ as

$\boldsymbol{u}=\boldsymbol{u}_{\perp}+u_{z} \boldsymbol{e}_{z}, \quad \boldsymbol{b}=\boldsymbol{b}_{\perp}+b_{z} \boldsymbol{e}_{z}$

we can reduce Eqs. (1)-(4) to

$\rho_{0} \frac{\mathrm{d} u_{z}}{\mathrm{~d} z}-\mathrm{i}\left(\omega \rho-\rho_{0} \boldsymbol{k} \cdot \boldsymbol{u}_{\perp}\right)=0$,

$\omega \boldsymbol{u}_{\perp}=\frac{\boldsymbol{k} P}{\rho_{0}}-\frac{c_{\mathrm{A}}^{2} \boldsymbol{b}_{\perp}(\boldsymbol{k} \cdot \boldsymbol{B})}{B^{2}}$,

$\omega u_{z}=-\frac{\mathrm{i}}{\rho_{0}} \frac{\mathrm{d} P}{\mathrm{~d} z}-\frac{c_{\mathrm{A}}^{2} b_{z}(\boldsymbol{k} \cdot \boldsymbol{B})}{B^{2}}-\frac{\mathrm{i} g \rho}{\rho_{0}}$,

$\omega \boldsymbol{b}_{\perp}=\boldsymbol{B}(\boldsymbol{k} \cdot \boldsymbol{u})-\boldsymbol{u}_{\perp}(\boldsymbol{k} \cdot \boldsymbol{B})-\mathrm{i} \boldsymbol{B} \frac{\mathrm{d} u_{z}}{\mathrm{~d} z}-\frac{\mathrm{i} \eta_{\mathrm{A}}}{B^{2}}\left\{(\boldsymbol{k} \cdot \boldsymbol{B})^{2} \boldsymbol{b}_{\perp}\right.$

$$
\left.-\boldsymbol{B} \frac{\mathrm{d}^{2}(\boldsymbol{b} \cdot \boldsymbol{B})}{\mathrm{d} z^{2}}+(\boldsymbol{b} \cdot \boldsymbol{B})\left[k^{2} \boldsymbol{B}-\boldsymbol{k}(\boldsymbol{k} \cdot \boldsymbol{B})\right]\right\},
$$

$\omega b_{z}=(\boldsymbol{k} \cdot \boldsymbol{B})\left[-u_{z}+\frac{\eta_{\mathrm{A}}}{B^{2}} \boldsymbol{B} \cdot\left(\frac{\mathrm{d} \boldsymbol{b}}{\mathrm{d} z}-\mathrm{i} \boldsymbol{k} b_{z}\right)\right]$,

$p=c_{\mathrm{s}}^{2} \rho$,

where $c_{\mathrm{s}}$ is the sound speed defined by

$c_{\mathrm{s}}^{2}=\frac{\gamma p_{0}}{\rho_{0}}$.

When deriving Eqs. (19) and (20) we have used the equation $\nabla \cdot \boldsymbol{b}=0$.

The boundary condition (13) reduces to

$\llbracket g \rho_{0} u_{z}+\mathrm{i} \omega P \rrbracket=0 \quad$ at $z=0$.

The boundary condition (14) can be transformed into

$$
\begin{aligned}
\boldsymbol{k} \times \boldsymbol{b}_{\perp 2} & +\left(\boldsymbol{k} \times \boldsymbol{e}_{z}\right) b_{z 2}-\mathrm{i} \boldsymbol{e}_{z} \times \frac{\mathrm{d} \boldsymbol{b}_{\perp 2}}{\mathrm{~d} z}-\boldsymbol{e}_{z}\left[\boldsymbol{e}_{z} \cdot\left(\boldsymbol{k} \times \boldsymbol{b}_{\perp 2}\right)\right] \\
& -\frac{\boldsymbol{B}_{2}}{B_{2}^{2}}\left(\left[\boldsymbol{B}_{2} \cdot\left(\boldsymbol{k} \times \boldsymbol{e}_{z}\right)\right] b_{z 2}-\mathrm{i}\left(\boldsymbol{B}_{2} \times \boldsymbol{e}_{z}\right) \cdot \frac{\mathrm{d} \boldsymbol{b}_{\perp 2}}{\mathrm{~d} z}\right)=0 .
\end{aligned}
$$

It is straightforward to verify that the projections of the left-hand side of this equation on vectors $\boldsymbol{e}_{z}$ and $\boldsymbol{B}_{2}$ are equal to zero. Hence, the only non-trivial projection is on vector $\boldsymbol{e}_{z} \times \boldsymbol{B}_{2}$ and it becomes

$\boldsymbol{B}_{2} \cdot\left(\frac{\mathrm{d} \boldsymbol{b}_{2}}{\mathrm{~d} z}-\mathrm{i} \boldsymbol{k} b_{z 2}\right)=0 \quad$ at $\quad z=0$ 
Eliminating $\rho, \boldsymbol{u}_{\perp}$, and $\boldsymbol{b}_{\perp}$ from Eqs. (16)-(21) we obtain the system of equations for $u_{z}, b_{z}$, and $P$,

$$
\begin{gathered}
\left(g \frac{\mathrm{d}}{\mathrm{d} z}+\omega^{2}\right)\left(u_{z}+\frac{\boldsymbol{k} \cdot \boldsymbol{B}}{\mu_{0} \rho_{0} \omega} b_{z}\right)+\frac{\mathrm{i} \omega}{\rho_{0}}\left(\frac{\mathrm{d} P}{\mathrm{~d} z}+\frac{g k^{2}}{\omega^{2}} P\right)=0, \\
(\boldsymbol{k} \cdot \boldsymbol{B}) u_{z}+\omega b_{z}=\frac{\mathrm{i} \eta_{\mathrm{A}}(\boldsymbol{k} \cdot \boldsymbol{B})}{\omega c_{\mathrm{A}}^{2}}\left(\frac{\mathrm{d} \Psi}{\mathrm{d} z}-\frac{\omega(\boldsymbol{k} \cdot \boldsymbol{B})}{\mu_{0} \rho_{0}} b_{z}\right), \\
{\left[\omega^{2}\left(c_{\mathrm{A}}^{2}+c_{\mathrm{s}}^{2}\right)-\omega_{\mathrm{A}}^{2} c_{\mathrm{s}}^{2}-\mathrm{i} \omega c_{\mathrm{s}}^{2} \eta_{\mathrm{A}}\left(\frac{\mathrm{d}^{2}}{\mathrm{~d} z^{2}}-k^{2}\right)\right] \Psi=} \\
\frac{\mathrm{i} \omega}{\rho_{0}}\left(\omega_{\mathrm{A}}^{2} c_{\mathrm{s}}^{2}-\omega^{2} c_{\mathrm{A}}^{2}\right) P,
\end{gathered}
$$

where

$\Psi=c_{\mathrm{s}}^{2}\left(\frac{\mathrm{d} u_{z}}{\mathrm{~d} z}+\frac{\boldsymbol{k} \cdot \boldsymbol{B}}{\mu_{0} \rho_{0} \omega} \frac{\mathrm{d} b_{z}}{\mathrm{~d} z}\right)-\frac{\mathrm{i}\left(\omega^{2}-c_{\mathrm{s}}^{2} k^{2}\right)}{\rho_{0} \omega} P$,

and $\omega_{\mathrm{A}}$ is the Alfvén frequency defined by

$\omega_{\mathrm{A}}=\frac{\boldsymbol{k} \cdot \boldsymbol{B}}{\sqrt{\mu_{0} \rho_{0}}}$.

The boundary condition given by Eq. (25) reduces to

$$
\frac{\mathrm{d} \Psi_{2}}{\mathrm{~d} z}-\frac{\omega\left(\boldsymbol{k} \cdot \boldsymbol{B}_{2}\right)}{\mu_{0} \rho_{02}} b_{z 2}=0
$$

Finally, we require that perturbations should be evanescent in the transversal direction; therefore, we impose the condition that all perturbations tend to zero as $|z| \rightarrow \infty$.

Eliminating all variables in favour of $P$ in the system of Eqs. (26)-(29) we can obtain the differential equation for $P$,

$$
\begin{gathered}
{\left[\omega^{2}\left(c_{\mathrm{A}}^{2}+c_{\mathrm{s}}^{2}\right)-\omega_{\mathrm{A}}^{2} c_{\mathrm{s}}^{2}-\mathrm{i} \omega c_{\mathrm{s}}^{2} \eta_{\mathrm{A}}\left(\frac{\mathrm{d}^{2}}{\mathrm{~d} z^{2}}-k^{2}\right)\right]\left(c_{\mathrm{s}}^{2} \frac{\mathrm{d}^{2} P}{\mathrm{~d} z^{2}}+g \frac{\mathrm{d} P}{\mathrm{~d} z}\right.} \\
\left.+\left(\omega^{2}-k^{2} c_{\mathrm{s}}^{2}\right) P\right)=\left(\omega^{2} c_{\mathrm{A}}^{2}-\omega_{\mathrm{A}}^{2} c_{\mathrm{s}}^{2}\right)\left(g \frac{\mathrm{d} P}{\mathrm{~d} z}+\omega^{2} P\right) .
\end{gathered}
$$

In order to be able to derive a dispersion relation, we would also need the equations that relate the variables $u_{z}, b_{z}$, and $\Psi$ with $P$, and they are

$$
\begin{aligned}
& g \frac{\mathrm{d} u_{z}}{\mathrm{~d} z}+\omega^{2} u_{z}=\frac{-1}{\rho_{0}\left[c_{\mathrm{A}}^{2}\left(\omega^{2}-\omega_{\mathrm{A}}^{2}\right)+\mathrm{i} \omega \omega_{\mathrm{A}}^{2} \eta_{\mathrm{A}}\right]} \\
& \times\left[\omega_{\mathrm{A}}^{2} \eta_{\mathrm{A}}\left(c_{\mathrm{s}}^{2} \frac{\mathrm{d}^{3} P}{\mathrm{~d} z^{3}}+g \frac{\mathrm{d}^{2} P}{\mathrm{~d} z^{2}}\right)+\left(\mathrm{i} c_{\mathrm{A}}^{2} \omega^{3}\right.\right. \\
&\left.\left.-\eta_{\mathrm{A}} \omega_{\mathrm{A}}^{2} k^{2} c_{\mathrm{s}}^{2}\right) \frac{\mathrm{d} P}{\mathrm{~d} z}+g k^{2}\left(\mathrm{i} \omega c_{\mathrm{A}}^{2}-\omega_{\mathrm{A}}^{2} \eta_{\mathrm{A}}\right) P\right] \\
& g \frac{\mathrm{d} b_{z}}{\mathrm{~d} z}+\omega^{2} b_{z}=\frac{\omega(\boldsymbol{k} \cdot \boldsymbol{B})}{\rho_{0}\left[c_{\mathrm{A}}^{2}\left(\omega^{2}-\omega_{\mathrm{A}}^{2}\right)+\mathrm{i} \omega \omega_{\mathrm{A}}^{2} \eta_{\mathrm{A}}\right]}\left(c_{\mathrm{s}}^{2} \eta_{\mathrm{A}} \frac{\mathrm{d}^{3} P}{\mathrm{~d} z^{3}}\right. \\
&\left.\quad+g \eta_{\mathrm{A}} \frac{\mathrm{d}^{2} P}{\mathrm{~d} z^{2}}+\left[i c_{\mathrm{A}}^{2} \omega+\eta_{\mathrm{A}}\left(\omega^{2}-k^{2} c_{\mathrm{s}}^{2}\right)\right] \frac{\mathrm{d} P}{\mathrm{~d} z}+\frac{\mathrm{i} g k^{2} c_{\mathrm{A}}^{2}}{\omega} P\right) \\
& g \frac{\mathrm{d} \Psi}{\mathrm{d} z}+\omega^{2} \Psi=-\frac{\mathrm{i} \omega}{\rho_{0}}\left(c_{\mathrm{s}}^{2} \frac{\mathrm{d}^{2} P}{\mathrm{~d} z^{2}}+g \frac{\mathrm{d} P}{\mathrm{~d} z}+\left(\omega^{2}-k^{2} c_{\mathrm{s}}^{2}\right) P\right)
\end{aligned}
$$

Assuming that in each region the dependence of the total pressure on $z$ varies as $\sim \mathrm{e}^{\lambda z}$ we can determine the characteristic equation corresponding to Eq. (32) as

$$
\begin{aligned}
\omega \eta_{\mathrm{A}} c_{\mathrm{s}}^{2} \lambda^{4} & +\omega g \eta_{\mathrm{A}} \lambda^{3}+\left[\mathrm{i} \omega^{2}\left(c_{\mathrm{A}}^{2}+c_{\mathrm{s}}^{2}\right)-\mathrm{i} \omega_{\mathrm{A}}^{2} c_{\mathrm{s}}^{2}\right. \\
& \left.+\omega \eta_{\mathrm{A}}\left(\omega^{2}-2 k^{2} c_{\mathrm{s}}^{2}\right)\right] \lambda^{2}+\omega g \lambda\left(\mathrm{i} \omega-\eta_{\mathrm{A}} k^{2}\right) \\
& +\mathrm{i}\left[\omega^{4}-\omega^{2} k^{2}\left(c_{\mathrm{A}}^{2}+c_{\mathrm{s}}^{2}\right)+k^{2} \omega_{\mathrm{A}}^{2} c_{\mathrm{s}}^{2}\right] \\
& -\omega k^{2} \eta_{\mathrm{A}}\left(\omega^{2}-k^{2} c_{\mathrm{s}}^{2}\right)=0
\end{aligned}
$$

Obviously the sign of the parameter $\lambda$ will be chosen in such a way that the evanescence of the perturbation in the transversal direction is insured. In what follows we determine solutions to Eq. (36) in both regions and then match them at the interface.

\subsection{Solution in the lower region}

In the lower region $(z<0) \eta_{\mathrm{A}}=0$, therefore, Eq. (36) reduces to

$$
\begin{aligned}
{\left[\omega ^ { 2 } \left(c_{\mathrm{A} 1}^{2}+\right.\right.} & \left.\left.c_{\mathrm{s} 1}^{2}\right)-\omega_{\mathrm{A} 1}^{2} c_{\mathrm{s} 1}^{2}\right] \lambda^{2}+\omega^{2} g \lambda \\
& +\omega^{4}-\omega^{2} k^{2}\left(c_{\mathrm{A} 1}^{2}+c_{\mathrm{s} 1}^{2}\right)+k^{2} \omega_{\mathrm{A} 1}^{2} c_{\mathrm{s} 1}^{2}=0 .
\end{aligned}
$$

We assume that this quadratic equation has exactly one root with the positive real part. We denote this root as $\lambda_{1}$. Under this assumption it follows from Eqs. (33) and (34) that the solution decaying as $z \rightarrow-\infty$ is given by

$$
\begin{aligned}
& P_{1}=A_{1} \mathrm{e}^{\lambda_{1} z}, \quad u_{z 1}=-\frac{\mathrm{i} \omega A_{1} \mathrm{e}^{\lambda_{1} z}\left(\omega^{2} \lambda_{1}+g k^{2}\right)}{\rho_{1}\left(g \lambda_{1}+\omega^{2}\right)\left(\omega^{2}-\omega_{\mathrm{A} 1}^{2}\right)}, \\
& b_{z 1}=\frac{\mathrm{i} A_{1} \mathrm{e}^{\lambda_{1} z}\left(\boldsymbol{k} \cdot \boldsymbol{B}_{1}\right)\left(\omega^{2} \lambda_{1}+g k^{2}\right)}{\rho_{1}\left(g \lambda_{1}+\omega^{2}\right)\left(\omega^{2}-\omega_{\mathrm{A} 1}^{2}\right)},
\end{aligned}
$$

where $A_{1}$ is an arbitrary constant.

\subsection{Solution in the upper region}

In the upper region $(z>0)$ we take into account the ambipolar diffusion, i.e. $\eta_{\mathrm{A}} \neq 0$. Now we assume that in this case Eq. (36) has exactly two roots with negative real parts. We denote these roots as $-\lambda_{2}$ and $-\lambda_{3}$. The general solution to Eq. (32) tending to zero as $z \rightarrow \infty$ is

$P_{2}=A_{2} \mathrm{e}^{-\lambda_{2} z}+A_{3} \mathrm{e}^{-\lambda_{3} z}$,

where $A_{2}$ and $A_{3}$ are arbitrary constants. Then it follows from Eqs. (33)-(35) that

$$
\begin{aligned}
u_{z 2}= & \frac{1}{\rho_{2} W} \sum_{j=2}^{3} \frac{A_{j} \mathrm{e}^{-\lambda_{j} z}}{\omega^{2}-g \lambda_{j}}\left[\eta_{\mathrm{A}} \omega_{\mathrm{A} 2}^{2} \lambda_{j}^{2}\left(c_{\mathrm{s} 2}^{2} \lambda_{j}-g\right)+\lambda_{j}\left(\mathrm{i} c_{\mathrm{A} 2}^{2} \omega^{3}\right.\right. \\
& \left.\left.-\eta_{\mathrm{A}} \omega_{\mathrm{A} 2}^{2} k^{2} c_{\mathrm{s} 2}^{2}\right)-g k^{2}\left(\mathrm{i} \omega c_{\mathrm{A} 2}^{2}-\eta_{\mathrm{A}} \omega_{\mathrm{A} 2}^{2}\right)\right], \\
b_{z 2}= & \frac{\boldsymbol{k} \cdot \boldsymbol{B}_{2}}{\rho_{2} W} \sum_{j=2}^{3} \frac{A_{j} \mathrm{e}^{-\lambda_{j} z}}{g \lambda_{j}-\omega^{2}}\left\{\eta_{\mathrm{A}} \omega \lambda_{j}^{2}\left(c_{\mathrm{s} 2}^{2} \lambda_{j}-g\right)\right. \\
& \left.+\lambda_{j} \omega\left[i c_{\mathrm{A} 2}^{2} \omega+\eta_{\mathrm{A}}\left(\omega^{2}-k^{2} c_{\mathrm{s} 2}^{2}\right)\right]-\mathrm{i} g k^{2} c_{\mathrm{A} 2}^{2}\right\}, \\
\Psi_{2}= & \frac{\mathrm{i} \omega}{\rho_{2}} \sum_{j=2}^{3} \frac{A_{j} \mathrm{e}^{-\lambda_{j} z}\left(c_{\mathrm{s} 2}^{2} \lambda_{j}^{2}-g \lambda_{j}+\omega^{2}-k^{2} c_{\mathrm{s} 2}^{2}\right)}{g \lambda_{j}-\omega^{2}},
\end{aligned}
$$

where

$W=c_{\mathrm{A} 2}^{2}\left(\omega^{2}-\omega_{\mathrm{A} 2}^{2}\right)+i \omega \omega_{\mathrm{A} 2}^{2} \eta_{\mathrm{A}}$. 


\subsection{Matching solutions}

At this stage it is convenient to introduce the dimensionless variables

$\zeta=\frac{\rho_{2}}{\rho_{1}}, \quad \kappa=\frac{k c_{\mathrm{A} 2}^{2}}{g}, \quad \sigma=-\frac{\mathrm{i} \omega c_{\mathrm{A} 2}}{g}, \quad \chi=\frac{B_{1}^{2}}{B_{2}^{2}}, \quad v=\frac{g \eta_{\mathrm{A}}}{c_{\mathrm{A} 2}^{3}}$,

$\beta_{1,2}=\frac{c_{\mathrm{s} 1,2}^{2}}{c_{\mathrm{A} 1,2}^{2}}, \quad \Lambda_{1}=\frac{\lambda_{1} c_{\mathrm{A} 2}^{2}}{g}, \quad \Lambda_{2,3}=\frac{\lambda_{2,3} c_{\mathrm{A} 2}^{2}}{g}$.

It follows from Eq. (9) that due to the continuity of the equilibrium total pressure we can write

$\chi=\frac{2 \beta_{2}+\gamma}{2 \beta_{1}+\gamma}$

In the dimensionless variables Eqs. (36) and (37) reduce to

$$
\begin{aligned}
& v \sigma \beta_{2} \Lambda_{j}^{4}-v \sigma \Lambda_{j}^{3}- {\left[\sigma^{2}\left(1+\beta_{2}\right)+v \sigma\left(\sigma^{2}+2 \kappa^{2} \beta_{2}\right)\right.} \\
&\left.+\kappa^{2} \beta_{2} \cos ^{2} \phi\right] \Lambda_{j}^{2}+ \sigma\left(\sigma+\kappa^{2} v\right) \Lambda_{j}+\sigma^{4}+\sigma^{2} \kappa^{2}\left(1+\beta_{2}\right) \\
&+ \kappa^{4} \beta_{2} \cos ^{2} \phi+\sigma v \kappa^{2}\left(\sigma^{2}+\kappa^{2} \beta_{2}\right)=0 \\
& \chi \zeta\left[\sigma^{2}\left(1+\beta_{1}\right)+\beta_{1} \chi \zeta \kappa^{2} \cos ^{2}(\phi-\alpha)\right] \Lambda_{1}^{2}+\sigma^{2} \Lambda_{1} \\
&-\sigma^{4}-\chi \zeta \sigma^{2} \kappa^{2}\left(1+\beta_{1}\right)-\beta_{1} \chi^{2} \zeta^{2} \kappa^{4} \cos ^{2}(\phi-\alpha)=0 .
\end{aligned}
$$

In Eq. (46) the index $j$ takes the values $j=2,3$. In what follows we only consider unstable modes and make an assumption that the value of $\sigma$ corresponding to these modes is real. Since the value of the dimensionless quantity $\sigma$ is positive, it immediately follows that Eq. (47) has exactly one real positive root. It is proved in Appendix A that, for $\sigma>0$, Eq. (46) has exactly two real positive roots.

To match the solutions in the lower and upper regions we substitute them in the boundary conditions Eqs. (10), (23), and (25). This yields

$$
\begin{aligned}
& \frac{\zeta \sigma A_{1}\left(\sigma^{2} \Lambda_{1}-\kappa^{2}\right)}{\left(\Lambda_{1}-\sigma^{2}\right)\left[\sigma^{2}+\chi \zeta \kappa^{2} \cos ^{2}(\phi-\alpha)\right]}-\frac{1}{\widetilde{W}} \sum_{j=2}^{3} \frac{A_{j}}{\sigma^{2}+\Lambda_{j}} \\
& \quad \times\left[v \Lambda_{j}^{2} \kappa^{2}\left(\beta_{2} \Lambda_{j}-1\right) \cos ^{2} \phi+\Lambda_{j}\left(\sigma^{3}-v \beta_{2} \kappa^{4} \cos ^{2} \phi\right)\right. \\
& \left.+\kappa^{2}\left(\sigma+v \kappa^{2} \cos ^{2} \phi\right)\right]=0, \\
& \frac{\sigma A_{1} U}{\left(\Lambda_{1}-\sigma^{2}\right)\left[\sigma^{2}+\chi \zeta \kappa^{2} \cos ^{2}(\phi-\alpha)\right]} \\
& -\frac{1}{\widetilde{W}} \sum_{j=2}^{3} \frac{A_{j}}{\sigma^{2}+\Lambda_{j}}\left\{v \kappa^{2} \Lambda_{j}^{2}\left(\beta_{2} \Lambda_{j}-1\right) \cos ^{2} \phi\right. \\
& -\Lambda_{j} \kappa^{2}\left[\sigma+v\left(\sigma^{2}+\beta_{2} \kappa^{2}\right)\right] \cos ^{2} \phi-v \kappa^{2}\left(\sigma^{4}-\kappa^{2}\right) \cos ^{2} \phi \\
& \left.-\sigma\left(\sigma^{4}+\sigma^{2} \kappa^{2} \cos ^{2} \phi-\kappa^{2}\right)\right\}=0, \\
& \sum_{j=2}^{3} \frac{A_{j} Q_{j}}{\sigma^{2}+\Lambda_{j}}=0,
\end{aligned}
$$

where we used the notation

$$
\begin{aligned}
\widetilde{W}= & \left(\sigma^{2}+\kappa^{2} \cos ^{2} \phi\right)+v \sigma \kappa^{2} \cos ^{2} \phi, \\
U= & \sigma^{4}-\kappa^{2}+\kappa^{2} \chi \zeta\left(\sigma^{2}-\Lambda_{1}\right) \cos ^{2}(\phi-\alpha), \\
Q_{j}= & \Lambda_{j}\left(\sigma^{2}+\kappa^{2} \cos ^{2} \phi\right)\left(\beta_{2} \Lambda_{j}^{2}-\Lambda_{j}-\beta_{2} \kappa^{2}\right) \\
& -\sigma^{4} \Lambda_{j}+\kappa^{4} \cos ^{2} \phi .
\end{aligned}
$$

The constant $A_{1}$ can be eliminated using Eqs. (48) and (49) to obtain

$\sum_{j=2}^{3} \frac{A_{j} S_{j}}{\sigma^{2}+\Lambda_{j}}=0$

where

$$
\begin{aligned}
S_{j}= & \zeta\left(\sigma^{2} \Lambda_{1}-\kappa^{2}\right)\left\{v \kappa^{2} \Lambda_{j}^{2}\left(\beta_{2} \Lambda_{j}-1\right) \cos ^{2} \phi\right. \\
& -\Lambda_{j} \kappa^{2}\left[\sigma+v\left(\sigma^{2}+\beta_{2} \kappa^{2}\right)\right] \cos ^{2} \phi \\
& \left.-v \kappa^{2}\left(\sigma^{4}-\kappa^{2}\right) \cos ^{2} \phi-\sigma\left(\sigma^{4}+\sigma^{2} \kappa^{2} \cos ^{2} \phi-\kappa^{2}\right)\right\} \\
& -U\left[v \Lambda_{j}^{2} \kappa^{2}\left(\beta_{2} \Lambda_{j}-1\right) \cos ^{2} \phi\right. \\
& \left.+\Lambda_{j}\left(\sigma^{3}-v \beta_{2} \kappa^{4} \cos ^{2} \phi\right)+\kappa^{2}\left(\sigma+v \kappa^{2} \cos ^{2} \phi\right)\right] .
\end{aligned}
$$

Equations (50) and (54) constitute the system of two linear homogeneous algebraic equations for $A_{2}$ and $A_{3}$. The condition of existence of non-trivial solutions to this system gives the dispersion equation

$Q_{2} S_{3}-Q_{3} S_{2}=0$.

Equations (46), (47), and (56) determine the dependence of $\sigma$ on the value of $\kappa$.

\section{Investigation of the dispersion equation}

\subsection{Analytical results}

\subsubsection{Critical wavenumber}

Ruderman (2017) studied the MRT stability of a magnetic interface in the approximation of ideal MHD. He showed that a normal mode is only unstable when the wavenumber is smaller than the critical wavenumber. This critical wavenumber is independent of the plasma $\beta$ and is the same as that obtained by Ruderman et al. (2014) in the approximation of incompressible plasma. We will now show that this result remains valid even when the ambipolar diffusion is taken into account, i.e. in partially ionised plasmas.

Since for unstable modes the value of $\sigma$ was assumed to be real, we obtain that $\sigma \rightarrow 0$ when $\kappa \rightarrow \kappa_{\mathrm{c}}$, where $\kappa_{\mathrm{c}}$ is the critical wavenumber in the dimensionless form. When $\sigma \rightarrow 0$ it follows from Eq. (47) that $\Lambda_{1}=\kappa+O\left(\sigma^{2}\right)$. It is easy to show that in this limit we can obtain from Eq. (46) that

$\Lambda_{2}=\kappa+O(\sigma), \quad \Lambda_{3}=\frac{\kappa \cos \phi}{\sqrt{v \sigma}}+O(1)$.

Substituting these results into Eqs. (53) and (55) yields

$Q_{2}=O\left(\sigma^{2}\right), \quad Q_{3}=O\left(\sigma^{-3 / 2}\right), \quad S_{3}=O\left(\sigma^{-3 / 2}\right)$,

$S_{2}=\sigma \kappa^{4}\left\{\zeta \kappa\left[\cos ^{2} \phi+\chi \cos ^{2}(\phi-\alpha)\right]-\zeta+1\right\}+O\left(\sigma^{2}\right)$.

Using these estimations we obtain from Eq. (56) that $S_{2} / \sigma \rightarrow 0$ as $\sigma \rightarrow 0$. As a result we arrive at

$\kappa_{\mathrm{c}}=\frac{\zeta-1}{\zeta\left[\cos ^{2} \phi+\chi \cos ^{2}(\phi-\alpha)\right]}$,

which is identical to the expression for $\kappa_{\mathrm{c}}$ obtained by Ruderman et al. (2014) and Ruderman (2017). A normal mode is unstable when $\kappa<\kappa_{\mathrm{c}}$ and stable otherwise. The above equation also means that there are particular values of angles (i.e. $\alpha=0, \phi=(2 n+1) \pi / 2)$ for which the critical wavenumber is infinity, i.e. all waves are unstable. This corresponds to the case of parallel magnetic fields at both sides. 


\subsubsection{Approximation of ideal MHD}

Next we show that the dispersion equation Eq. (56) reduces to the dispersion equation derived by Ruderman (2017) in the approximation of ideal MHD, i.e. when $v \rightarrow 0$. Let us introduce the same notation as in Ruderman (2017), in particular

$$
\begin{aligned}
& \Psi_{1}=\sigma^{2}+\chi \zeta \kappa^{2} \cos ^{2}(\phi-\alpha), \\
& \Psi_{2}=\sigma^{2}+\kappa^{2} \cos ^{2} \phi, \\
& \Phi_{1}=\sigma^{2}\left(\beta_{1}+1\right)+\beta_{1} \chi \zeta \kappa^{2} \cos ^{2}(\phi-\alpha), \\
& \Phi_{2}=\sigma^{2}\left(\beta_{2}+1\right)+\beta_{2} \kappa^{2} \cos ^{2} \phi, \\
& \Delta_{1}=\sigma^{4}+\chi \zeta \kappa^{2} \sigma^{2}\left(\beta_{1}+1\right)+\beta_{1} \chi^{2} \zeta^{2} \kappa^{4} \cos ^{2}(\phi-\alpha), \\
& \Delta_{2}=\sigma^{4}+\kappa^{2} \sigma^{2}\left(\beta_{2}+1\right)+\beta_{2} \kappa^{4} \cos ^{2} \phi .
\end{aligned}
$$

Then we obtain from Eqs. (46), and (47) that

$\Lambda_{1}=\frac{-\sigma^{2}+\sqrt{\sigma^{4}+4 \chi \zeta \Phi_{1} \Delta_{1}}}{2 \chi \zeta \Phi_{1}}$

$\Lambda_{2}=\frac{\sigma^{2}+\sqrt{\sigma^{4}+4 \Phi_{2} \Delta_{2}}}{2 \Phi_{2}}+O(v), \quad \Lambda_{3}=O\left(v^{-1 / 2}\right)$.

Using Eq. (63) we obtain the estimates $Q_{2}=O(1), Q_{3}=$ $O\left(v^{-3 / 2}\right), S_{2}=O(1)$, and $S_{3}=O\left(v^{-1 / 2}\right)$. Then it follows from Eq. (56) in the leading order approximation with respect to $v$ that $S_{2}=0$. Taking $v=0$ in this equation we obtain

$\zeta\left(\sigma^{2} \Lambda_{1}-\kappa^{2}\right)\left(\sigma^{2} \Psi_{2}+\kappa^{2} \Lambda_{2} \cos ^{2} \phi-\kappa^{2}\right)$

$\left(\sigma^{2} \Lambda_{2}+\kappa^{2}\right)\left[\sigma^{2} \Psi_{1}-\chi \zeta \kappa^{2} \Lambda_{1} \cos ^{2}(\phi-\alpha)-\kappa^{2}\right]=0$.

Multiplying this equation by

$\left[\chi \zeta \Psi_{1}\left(\sigma^{2} \Lambda_{1}+\kappa^{2}\right)+\sigma^{4}\right]\left[\Psi_{2}\left(\sigma^{2} \Lambda_{2}-\kappa^{2}\right)-\sigma^{4}\right]$,

and using the identities

$\left(\sigma^{2} \Lambda_{1}-\kappa^{2}\right)\left[\chi \zeta \Psi_{1}\left(\sigma^{2} \Lambda_{1}+\kappa^{2}\right)+\sigma^{4}\right]=\sigma^{4}\left(\Delta_{1}-\kappa^{2}\right)-\chi \zeta \kappa^{4} \Psi_{1}$,

$\left(\sigma^{2} \Lambda_{2}+\kappa^{2}\right)\left[\Psi_{2}\left(\sigma^{2} \Lambda_{1}+\kappa^{2}\right)-\sigma^{4}\right]=\sigma^{4}\left(\Delta_{2}-\kappa^{2}\right)-\kappa^{4} \Psi_{2}$,

we transform Eq. (64) to

$$
\begin{aligned}
& \zeta\left[\sigma^{4}\left(\Delta_{1}-\kappa^{2}\right)-\chi \zeta \kappa^{4} \Psi_{1}\right]\left\{\sigma^{2} \kappa^{2} \Phi_{2} \Lambda_{2}^{2} \cos ^{2} \phi\right. \\
& +\left[\sigma^{2} \Phi_{2}\left(\sigma^{2} \Psi_{2}-\kappa^{2}\right)-\left(\kappa^{2} \Phi_{2}+\sigma^{4}\right) \kappa^{2} \cos ^{2} \phi\right] \Lambda_{2} \\
& \left.-\left(\sigma^{2} \Psi_{2}-\kappa^{2}\right)\left(\kappa^{2} \Phi_{2}+\sigma^{4}\right)\right\}-\left[\sigma^{4}\left(\Delta_{2}-\kappa^{2}\right)-\kappa^{4} \Psi_{1}\right] \\
& \times\left\{\chi^{2} \zeta^{2} \sigma^{2} \kappa^{2} \Phi_{1} \Lambda_{1}^{2} \cos ^{2}(\phi-\alpha)-\chi \zeta\left[\sigma^{2} \Phi_{1}\left(\sigma^{2} \Psi_{1}-\kappa^{2}\right)\right.\right. \\
& \left.-\left(\chi \zeta \kappa^{2} \Phi_{2}+\sigma^{4}\right) \kappa^{2} \cos ^{2}(\phi-\alpha)\right] \Lambda_{1} \\
& \left.-\left(\sigma^{2} \Psi_{1}-\kappa^{2}\right)\left(\chi \zeta \kappa^{2} \Phi_{2}+\sigma^{4}\right)\right\}=0 .
\end{aligned}
$$

Now, with the aid of Eqs. (61)-(63), after long but straightforward calculation we reduce Eq. (65) to

$$
\begin{aligned}
F\left(\sigma, \beta_{1}, \beta_{2}\right) \equiv & \frac{\zeta \Psi_{2}}{\Delta_{2}}\left(\sigma^{2}+\sqrt{\sigma^{4}+4 \Delta_{2} \Phi_{2}}\right) \\
& -\frac{\Psi_{1}}{\Delta_{1}}\left(\sigma^{2}-\sqrt{\sigma^{4}+4 \chi \zeta \Delta_{1} \Phi_{1}}\right)=2 \zeta-2 .
\end{aligned}
$$

This is exactly the dispersion equation obtained by Ruderman (2017) in the approximation of ideal MHD. We verified numerically for a very wide range of parameter variation that, for $\kappa \in\left(0, \kappa_{\mathrm{c}}\right), F\left(\sigma, \beta_{1}, \beta_{2}\right)$ is a monotonically increasing function of $\sigma$ when $\sigma>0$. Since $F\left(\sigma, \beta_{1}, \beta_{2}\right) \rightarrow 2(\zeta-1)\left(\kappa / \kappa_{\mathrm{c}}\right)$ as $\sigma \rightarrow 0$, and $F\left(\sigma, \beta_{1}, \beta_{2}\right) \rightarrow \infty$ as $\sigma \rightarrow \infty$, we conclude that Eq. (66) has exactly one positive root when $\kappa<\kappa_{\mathrm{c}}$, and no positive roots when $\kappa>\kappa_{\mathrm{c}}$.

\subsubsection{Approximation of incompressible plasma}

Finally, we consider the approximation of incompressible plasma and take $\beta_{1}=\beta_{2}=\beta \rightarrow \infty$. Then it follows from Eqs. (46) and (47) that

$$
\begin{aligned}
& \Lambda_{1}=\kappa+O\left(\beta^{-1}\right), \\
& \Lambda_{2}=\kappa+\frac{\beta^{-1} \sigma^{2}\left(\sigma^{2}+\kappa\right)}{2 \kappa\left(\sigma^{2}+\kappa^{2} \cos ^{2} \phi\right)}+O\left(\beta^{-2}\right), \\
& \Lambda_{3}=\sqrt{\kappa^{2}+\frac{\sigma^{2}+\kappa^{2} \cos ^{2} \phi}{v \sigma}}+O\left(\beta^{-1}\right) .
\end{aligned}
$$

Using these results we obtain the estimates

$Q_{2}=O\left(\beta^{-1}\right), \quad Q_{3}=O(\beta), \quad S_{3}=O(\beta)$,

and the expression

$$
\begin{aligned}
S_{2}= & -\sigma \kappa\left(\sigma^{4}-\kappa^{2}\right)\left(1+\frac{v \sigma \kappa \cos ^{2} \phi}{\sigma^{2}-\kappa^{2} \cos ^{2} \phi}\right)\left\{(\zeta+1) \sigma^{2}\right. \\
& \left.-(\zeta-1) \kappa+\zeta \kappa^{2}\left[\cos ^{2} \phi+\chi \cos ^{2}(\phi-\alpha)\right]\right\} .
\end{aligned}
$$

It follows from Eqs. (68) and (69) that, in the leading order approximation with respect to $\beta$, the dispersion equation Eq. (56) reduces to $S_{2}=0$. Hence, in the approximation of incompressible plasma, the dispersion relation is given by

$\sigma^{2}=\frac{(\zeta-1) \kappa-\zeta \kappa^{2}\left[\cos ^{2} \phi+\chi \cos ^{2}(\phi-\alpha)\right]}{\zeta+1}$.

This relation is exactly the equation derived by Ruderman et al. (2014) and Ruderman (2017) in the approximation of ideal MHD and incompressible plasma. In the approximation of incompressible plasma, we see that the ambipolar diffusion does not affect the MRT instability.

\subsection{Numerical results}

In this section we are going to analyse numerically the general dispersion relation (56). The instability increment $\sigma$ depends on the dimensionless parameters $\zeta, \chi, \beta_{1}, \beta_{2}, \kappa, \phi, \alpha$, and $\nu$, of which only seven are independent because $\chi, \beta_{1}$, and $\beta_{2}$ are related via Eq. (45). The left-hand side of Eq. (56) is a periodic function of both $\phi$ and $\alpha$, and the period with respect to each of these two angles is $\pi$. In addition, the dispersion relation is invariant with respect to the substitution $\pi-\phi \rightarrow \phi$ and $\pi-\alpha \rightarrow \alpha$. This observation enables us to restrict the intervals of variation of these two angles to $0 \leq \phi<\pi$ and $0 \leq \alpha \leq \pi / 2$.

When solving the dispersion equation Eq. (56) numerically we consider $\zeta=100$ and $\chi=1$. Then it follows from Eq. (45) that $\beta_{1}=\beta_{2}=\beta$. We now estimate the dimensionless coefficient of ambipolar diffusion $v$. For typical values of the prominence plasma we take $\rho_{2}=10^{-10} \mathrm{~kg} \mathrm{~m}^{-3}, c_{\mathrm{A} 2}=100 \mathrm{~km} \mathrm{~s}^{-1}$, $g=274 \mathrm{~m} \mathrm{~s}^{-2}$, and the plasma temperature $10^{4} \mathrm{~K}$. Then, taking into account that the prominence plasma is only weakly ionised and $\xi_{n} \approx 1$, we obtain

$v \approx \frac{5 \times 10^{-6}}{1-\xi_{n}}$

The above relation shows that unless the plasma is extremely weakly ionised, $v \ll 1$. We calculated the dependence of the instability increment $\sigma$ on $\kappa$ for various values of $\beta, \alpha$, and $\phi$, and for $v=10^{-4}, 0.1$, and 1 , which corresponds to 
M. S. Ruderman et al.: Rayleigh-Taylor instabilities with sheared magnetic fields
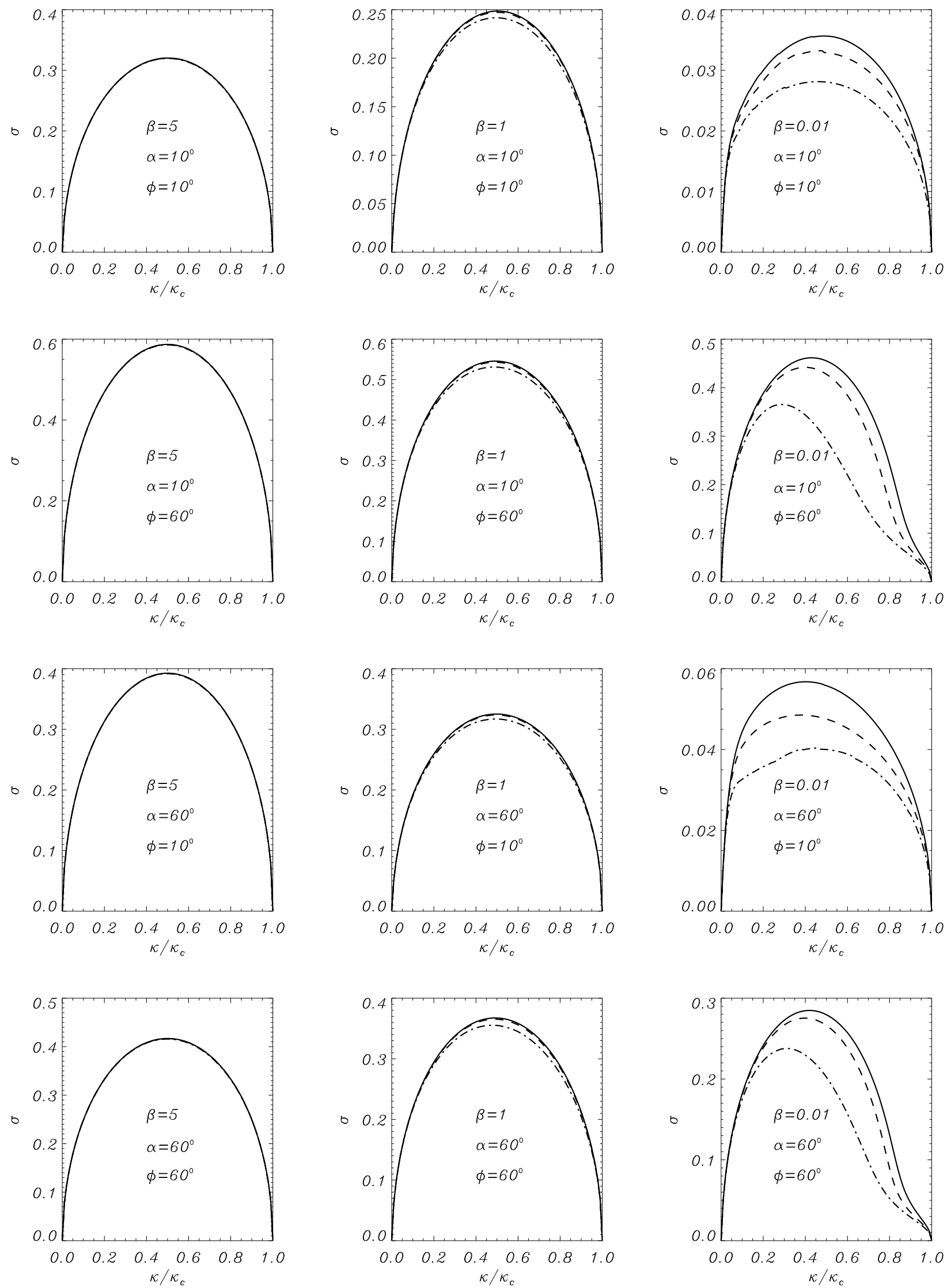

Fig. 2. Dependence of the dimensionless instability increment $\sigma$ on the ratio of the wavenumber to the critical wavenumber $\kappa / \kappa_{\mathrm{c}}$ for four particular values of the angles $\alpha$ and $\phi$. The left, middle, and right panels correspond to $\beta=5,1$, and 0.01 , respectively. The solid, dashed, and dash-dotted curves correspond to $v=10^{-4}, 0.1$, and 1 , respectively. In the left panels all the curves are indistinguishable. In the middle panels the solid and dashed curves are indistinguishable. 

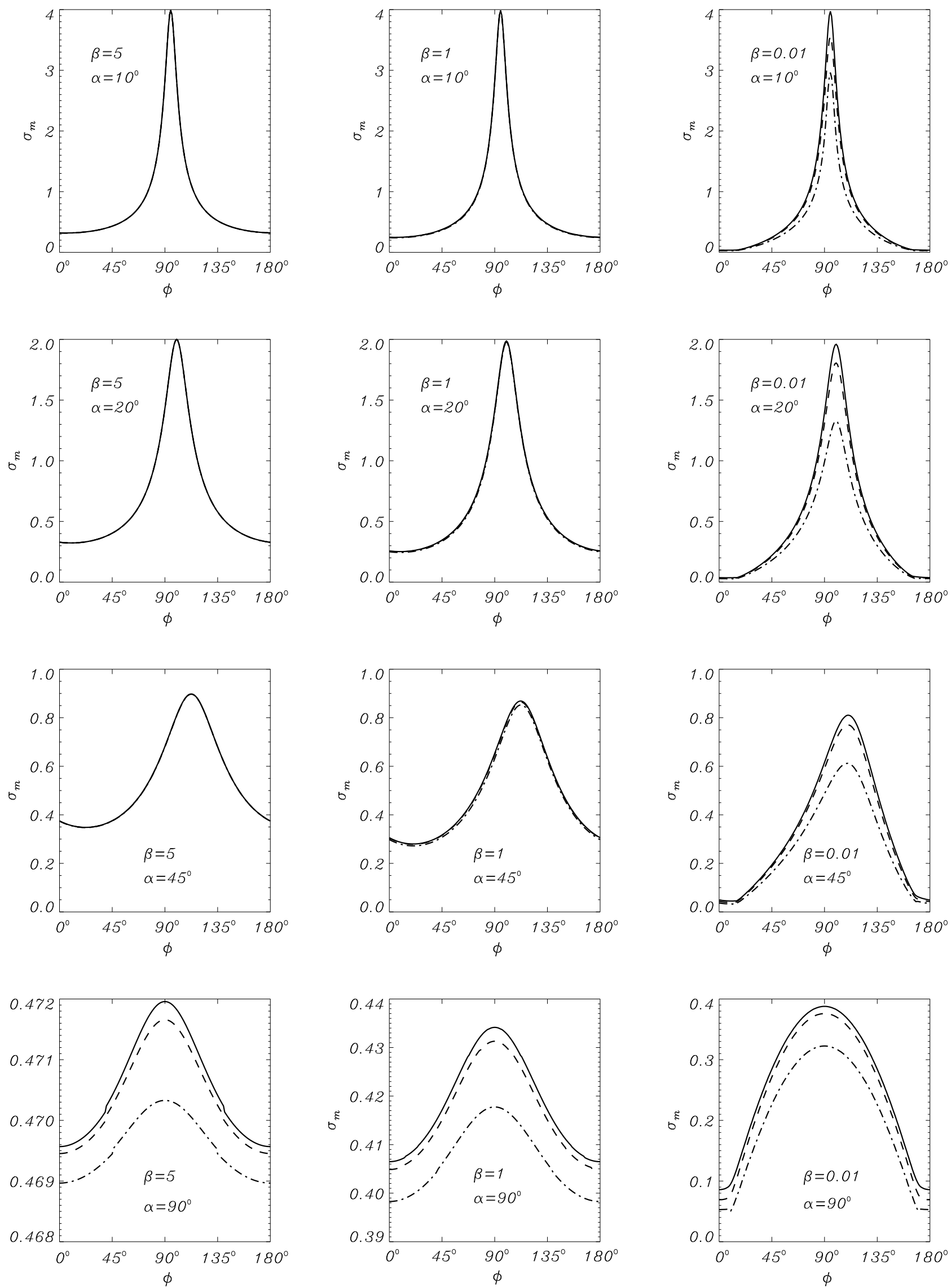

Fig. 3. Dependence of the maximum with respect to $\kappa$ of the dimensionless instability increment, $\sigma_{\mathrm{m}}$, on the angle $\phi$. The left, middle, and right panels correspond to $\beta=5,1$, and 0.01 , respectively. The solid, dashed, and dash-dotted curves correspond to $v=10^{-4}, 0.1$, and 1 , respectively. In the panels where one single curve is plotted all the curves are indistinguishable. 
$1-\xi_{n}=0.05,5 \times 10^{-5}$, and $5 \times 10^{-6}$. The results of this calculation are shown in Fig. 2. These plots show that when $\beta=5$, which is very close to the limit of incompressible plasma, the three curves are indistinguishable and the maximum instability increment is obtained for $\kappa / \kappa_{\mathrm{c}} \approx 0.5$. The result obtained in this case confirms the conclusion that, in the limit of incompressible plasma, the ambipolar diffusion does not affect the MRT instability. We verified that for all values of $\beta, \alpha$, and $\phi$ the solid curves corresponding to $v=10^{-4}$ are the same as those obtained in the approximation of ideal MHD. Hence, we can conclude that even when the prominence plasma is relatively weakly ionized with only one proton per 20 hydrogen atoms the effect of ambipolar diffusion on the appearance of the MRT instability at the interface between the prominence and corona is almost negligible.

In general, we see that the ambipolar diffusion reduces the instability increment and the maximum increment is obtained for smaller wavenumbers (larger wavelengths) as the number of neutrals is increased. For small values of plasma beta (as in the case of prominences) the decrease in the increment for $v=1$ can be up to $40 \%$. Nevertheless, even for these values of plasma beta, noticeable reduction in the instability increment occurs when the prominence plasma is extremely weakly ionised, with the ratio of number of neutral hydrogen atoms to the number of ions of the order of $10^{5}$.

We also study the dependence on $\phi$ of the maximum instability increment with respect to $\kappa$, and the dependence on $\alpha$ of the maximum instability increment with respect to $\kappa$ and $\phi$. These two quantities are defined by

$\sigma_{\mathrm{m}}=\max _{\kappa} \sigma, \quad \sigma_{\mathrm{M}}=\max _{\kappa, \phi} \sigma$.

The results of our calculations are shown in Figs. 3 and 4. In Fig. 3, we see that the effect of ambipolar diffusion becomes stronger when $\alpha$ increases and $\beta$ decreases. Again, a significant variation in the instability increment occurs for $\beta \ll 1$, i.e. for the prominence and upper chromospheric conditions. We also see that the maximum increment occurs when the wave vector is almost perpendicular to the ambient magnetic field in the prominence. When $\beta \ll 1$ the increment decreases with the increase in the number of neutrals in the plasma. The value of $\sigma_{\mathrm{m}}$ is almost independent of $\phi$ and close to 0.5 when $\alpha=90^{\circ}$ and $\beta=5$. This result agrees very well with the fact that, in the approximation of incompressible plasma, $\sigma_{\mathrm{m}}=0.5$ when $\alpha=90^{\circ}$. We also verified that the solid curves corresponding to $v=10^{-4}$ almost coincide with the similar curves obtained by Ruderman (2017) in the approximation of ideal MHD.

We verified that the solid curves in Fig. 4 corresponding to $v=10^{-4}$ coincide with the similar curves obtained by Ruderman (2017) in the approximation of ideal MHD. Figure 4 also reveals that the ambipolar diffusion only affects the maximum growth rate $\sigma_{\mathrm{M}}$ when the plasma beta is very small and, in addition, the prominence plasma is extremely weakly ionised. This conclusion is important for applications to the prominence seismology. Following the assumption by Terradas et al. (2012) that the prominence disappearance evident from the observational results reported by Okamoto et al. (2007) was caused by the MRT instability, Ruderman et al. (2014) estimated the shear angle $\alpha$ between the magnetic field in the prominence and in the surrounding coronal plasma. When doing so they used the results obtained in the approximation of incompressible plasma. Ruderman (2017) extended this analysis to include the plasma compressibility and also applied it to observations of the MRT instability in prominences reported by Ryutova et al. (2010). He found that the account of plasma compressibility
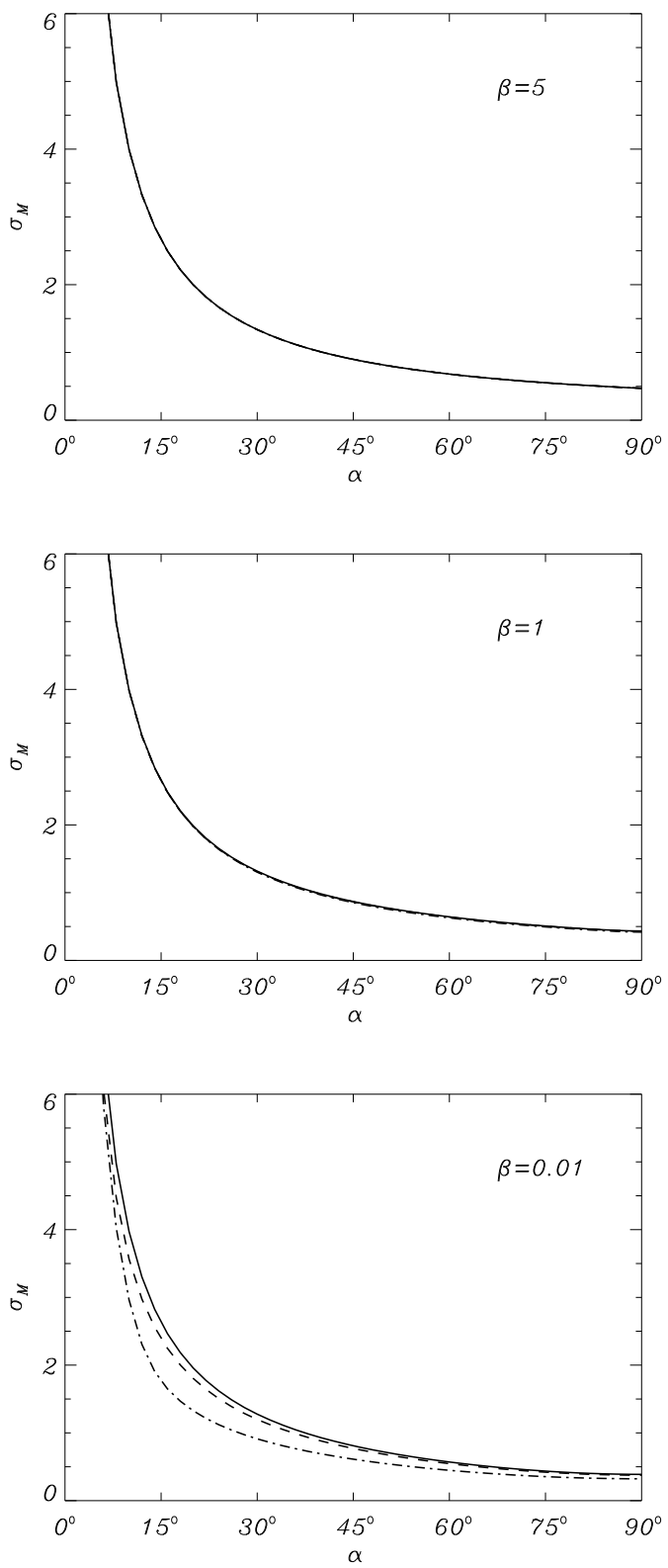

Fig. 4. Dependence of the maximum with respect to $\kappa$ and $\phi$ of the dimensionless instability increment, $\sigma_{\mathrm{M}}$, on the angle $\alpha$. The upper, middle, and lower panels correspond to $\beta=5,1$, and 0.01 , respectively. The solid, dashed, and dash-dotted curves correspond to $v=10^{-4}, 0.1$, and 1 , respectively. In the upper and middle panels all the curves are indistinguishable.

does not affect the estimates of $\alpha$. On the basis of the results obtained in this article we can make the same conclusion about the effect of ambipolar diffusion. The values of $\sigma_{M}$ in Fig. 4 do not reach a maximum value, instead their evolution towards smaller shear angle was cut because $\sigma_{\mathrm{M}} \rightarrow \infty$ as $\alpha \rightarrow 0$.

\section{Conclusions}

The present study deals with the problem of the magnetic Rayleigh-Taylor instability in partially ionised compressible plasmas in the presence of magnetic shear, meaning that the equilibrium magnetic field has different directions below and above the magnetic interface. The dynamics of the plasma was described within the framework of single-fluid MHD, and the effect of partial ionisation appeared in the induction equation in 
the form of ambipolar diffusion. The magnitude of the dissipative coefficient is determined by the amount of neutrals in the fluid.

First of all, our analysis demonstrated that the MRT instability only appears for wavenumbers that are smaller than a critical cut-off value. This value is determined by the density and magnetic field contrasts between the two regions, by the angle between the wave vector of a perturbation and the equilibrium magnetic field, and by the degree of magnetic shear. However, this critical wavenumber is independent of the plasma beta and ionisation degree of the plasma. Hence, fully and partially ionised plasmas have the same critical wavenumber. We need to mention here that the existence of the critical wavenumber is very much connected to the equilibrium chosen for our model. Díaz et al. (2014) and Khomenko et al. (2014) studied the MRT instability of a magnetic interface with the plasma only partially ionised at both sides. They found that for such an equilibrium there is no critical wavenumber meaning that perturbations with any wavenumber are unstable. Hence, the existence of the critical wavenumber is related to the assumption that the plasma is fully ionised at one side of the magnetic interface.

In the incompressible limit the ambipolar diffusion does not affect the evolution of the instability. This result is further confirmed by our numerical investigation for high values of plasma beta. When the plasma beta is large the curves showing the dependence of the maximum increment $\sigma_{\mathrm{M}}$ on the magnetic shear angle $\alpha$ and corresponding to different degrees of ionisation practically coincide (see Fig. 4). Our investigation showed that a significant dependence of the maximum instability increment on the ionisation degree only occurs in the $\beta \ll 1$ regime, where the decrease in the increment can be as large as $40 \%$ for the values of parameters considered here.

There is a simple physical explanation why the ambipolar diffusion does not affect the maximum increment of the MRT instability in the approximation of incompressible plasma corresponding to $\beta \rightarrow \infty$. Ambipolar diffusion appears because the neutrals and ions have different velocities. This velocity difference, in turn, appears because the Lorentz force affects the motion of ions and does not affect the motion of neutrals. In the limit of $\beta \rightarrow \infty$ the Lorentz force is negligible in comparison with the pressure gradient. As a result, the ions and neutrals move with the same velocity and the effect of ambipolar diffusion disappears. When $\beta$ is finite but large the velocities of ions and neutrals do not coincide; however, their difference is very small and, consequently, the effect of ambipolar diffusion is also very small. Our calculations show that this effect is actually very small even when $\beta$ is of the order of unity. It only becomes substantial when $\beta \ll 1$.
The value of $\beta$ also affects the maximum value of the increment; this maximum value decreases when $\beta$ decreases while the values of propagation angle and magnetic shear are fixed. The increment takes its maximum when the wave vector of a perturbation is almost perpendicular to the magnetic field in the dense plasma. It is only affected by the ionisation degree when this degree is extremely small and, in addition, $\beta$ is small.

We note that in the numerical investigation of the dispersion equation we always took $\beta_{1}=\beta_{2}=\beta$. If we make a viable assumption that $\beta_{2} \gtrsim 1$ when $\beta_{1} \gtrsim 1$, then ambipolar diffusion does not affect the maximum instability increment for any values of $\beta_{2} / \beta_{1}$. On the other hand, the maximum increment becomes independent of $\beta_{1}$ and $\beta_{2}$ when both these quantities are smaller than or of the order of 0.01 . For these small values of $\beta_{1}$ and $\beta_{2}$ it is very nearly equal to its limiting value obtained when $\beta_{1} \rightarrow 0$ and $\beta_{2} \rightarrow 0$.

Acknowledgements. This study was initiated when M.S.R. visited the Instituto de Astrofísica de Canarias (IAC). He thanks IAC for their warm hospitality. The authors acknowledge the financial support by the Leverhulme Trust (IN2014-016). M.S.R. also acknowledges the support by STFC. I.B. was partly supported by a grant of the Ministry of National Education and Scientific Research, RDI Programme for Space Technology and Advanced Research - STAR, project number 181/20.07.2017.

\section{References}

Bommier, V., Landi Degl'Innocenti, E., Leroy, J. L., \& Sahal-Brechot, S. 1994, Sol. Phys., 154, 231

Bucciantini, N., Amato, E., Bandiera, R., Blondin, J. M., \& Del Zanna, L. 2004, A\&A, 423, 253

Díaz, A. J., Khomenko, E., \& Collados, M. 2014, A\&A, 564, A97 Hilier, A., Isobe, H., Shibata, K., \& Berger, T. 2011, ApJ, 736, L1

Hilier, A., Berger, T., Isobe, H., \& Shibata, K. 2012a, ApJ, 746, 120

Hilier, A., Isobe, H., Shibata, K., \& Berger, T. 2012b, ApJ, 756, 110 Isobe, H., Miyagoshi, T., Shibata, K., \& Yokoyama, T. 2005, Nature, 434, 478 Isobe, H., Miyagoshi, T., Shibata, K., \& Yokoyama, T. 2006, PASJ, 58, 423 Jones, T. W., \& De Young, D. S. 2005, ApJ, 624, 586

Jun, B.-I., \& Norman, M. L. 1996, ApJ, 472, 245

Jun, B.-I., Norman, M. L., \& Stone, J. M. 1995, ApJ, 453, 332

Khomenko, E., Díaz, A. J., de Vicente, A., Collados, M., \& Luna, M. 2014, A\&A, 565, A45

Leroy, J. L., Bommier, V., \& Sahal-Brechot, S. 1984, A\&A, 131, 33

Okamoto, T. J., Tsuneta, S., Berger, T. E., et al. 2007, Science, 318, 1577

O’Neil, M., Alouani Bibi, F., Toth, J., et al. 2009, Nature, 462, 1036

Robinson, D., Dursi, L. J., Ricker, P. M., et al. 2004, ApJ, 601, 621

Ruderman, M. S. 2015, A\&A, 580, A37

Ruderman, M. S. 2017, Sol. Phys., 292, 47

Ruderman, M. S., Terradas, J., \& Ballester, J. L. 2014, ApJ, 785, A110

Ryutova, M. P., Berger, T., Frank, Z., Tarbell, T., \& Title, A. 2010, Sol. Phys., 267, 75

Terradas, J., Oliver, R., \& Ballester, J. L. 2012, A\&A, 541, A102 


\section{Appendix A: Investigation of Eq. (46)}

In this section we show that Eq. (46) has exactly two positive roots when $\sigma>0$. We rewrite Eq. (46) as

$f_{1}(\Lambda)=f_{2}(\Lambda)$

where

$$
\begin{aligned}
f_{1}(\Lambda)= & v \sigma\left(\Lambda^{2}-\kappa^{2}\right)\left(\beta_{2} \Lambda^{2}-\Lambda-\sigma^{2}-\beta_{2} \kappa^{2}\right), \\
f_{2}(\Lambda)= & {\left[\sigma^{2}\left(1+\beta_{2}\right)+\beta_{2} \kappa^{2} \cos ^{2} \phi\right] \Lambda^{2} } \\
& -\sigma^{2} \Lambda-\sigma^{4}-\sigma^{2} \kappa^{2}\left(1+\beta_{2}\right)-\beta_{2} \kappa^{4} \cos ^{2} \phi,
\end{aligned}
$$

and $\Lambda=\Lambda_{j}$. It is straightforward to see that the equation $f_{1}(\Lambda)=0$ has exactly two positive and two negative roots, and the equation $f_{2}(\Lambda)=0$ has exactly one positive and one negative root. Typical graphs of functions $f_{1}(\Lambda)$ and $f_{2}(\Lambda)$ are shown in Fig. A.1. We note that the mutual positions of negative zeros of functions $f_{1}(\Lambda)$ and $f_{2}(\Lambda)$ can be different from those shown in Fig. A.1. However, this fact is not important because we are only interested in the positive roots of Eq. (46). The smaller positive zero of function $f_{1}(\Lambda)$ is $\kappa$, while the larger positive zero is

$\Lambda_{0}=\frac{1+\sqrt{1+4 \beta_{2}\left(\sigma^{2}+\beta_{2} \kappa^{2}\right)}}{2 \beta_{2}}$.

We denote the positive zero of function $f_{1}(\Lambda)$ as $\Lambda_{+}$and prove that $\kappa<\Lambda_{+}<\Lambda_{0}$. We have $f_{2}(\kappa)=-\sigma^{2} \kappa<0$, which implies

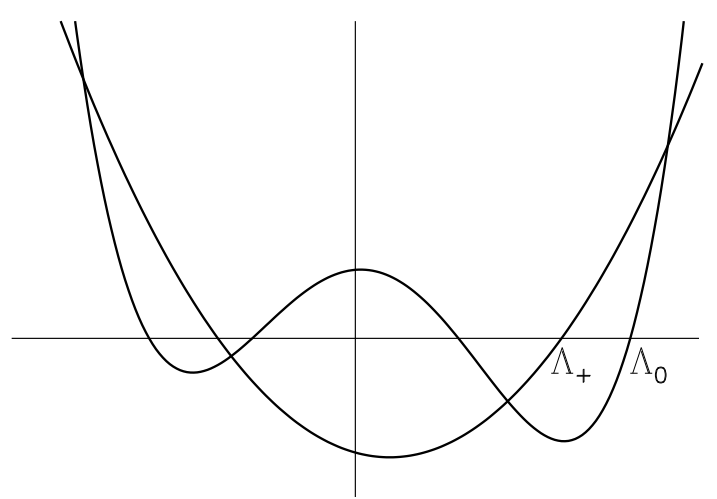

Fig. A.1. Graphical investigation of Eq. (A.1). The curve with four zeros is the graph of function $f_{1}(\Lambda)$, while the curve with two zeros is the graph of function $f_{2}(\Lambda)$.

that $\kappa<\Lambda_{+}$. After straightforward calculation we obtain that the inequality $f_{2}\left(\Lambda_{0}\right)>0$ is equivalent to the obvious inequality

$$
\sqrt{1+4 \beta_{2}\left(\sigma^{2}+\beta_{2} \kappa^{2}\right)}+1+2 \beta_{2} \sigma^{2}>0 .
$$

The inequality $f_{2}\left(\Lambda_{0}\right)>0$ implies that $\Lambda_{+}<\Lambda_{0}$. This inequality implies that the graphs of functions $f_{1}(\Lambda)$ and $f_{2}(\Lambda)$ have two points of intersection for $\Lambda>0$ meaning that Eq. (46) has exactly two positive roots. 